\title{
ISSUES IN MEDICINE Moral perspectives on stimulant use by healthy students
}

\author{
C Verster, A A van Niekerk
}

Chris Verster is a senior lecturer in the Department of Psychiatry at Stellenbosch University, where he also serves on the Health Research Ethics Committee. He is a consultant psychiatrist at Stikland Hospital, Bellville, Western Cape. Anton A van Niekerk is professor of philosophy and bioethics and director of the Centre for Applied Ethics at Stellenbosch University. He is chair of the Board of Directors of the Ethics Institute of South Africa and a member of the Ethics Committee of the Medical Research Council.

Corresponding author: C Verster (chrisv@sun.ac.za)

The quest for enhancement has been part of human culture for thousands of years. Progress in scientific developments and especially in medical science has enabled previously unimaginable advances to be employed in endeavours to improve human functioning in its various forms. Previously enhancement focused on aspects such as prolonging life, improving the immune system or cosmetic enhancements. Cognitive enhancement is currently receiving substantial attention. The use of stimulants such as methylphenidate, especially among students at tertiary institutions aiming to enhance their cognitive abilities, has raised concerns, ranging from safety issues and the risk of drug abuse to moral issues relating to the broader context of enhancement. We consider arguments used to debate both the promotion of enhancement therapies and the restriction and possibly even prevention of their use.

S Afr Med J 2012;102(12):909-911. DOI:10.7196/SAMJ.6090

In our world there is a continuous drive to be better, faster, stronger, prettier or more intelligent. We want to live longer, fight disease more effectively, and prevent disease wherever possible. Improved quality of life is a universal human endeavour.

The concept of biomedical enhancement may be defined as follows: 'a deliberate intervention, applying biomedical science, which aims to improve an existing capacity that most or all normal human beings typically have, or to create a new capacity, by acting directly on the body or brain.' ${ }^{1}$

Enhancement therapies are easily justified in those suffering from disabilities, but their use in healthy subjects, with the aim of improving 'normal' functioning, raises ethical issues.

There have been reports over the past few decades about uses for stimulants, such as methylphenidate, for which they are not traditionally indicated and for which these drugs are not registered. South African lay press reports claim that the use of methylphenidate, especially by healthy students with no previous or current diagnosis of attention deficit hyperactivity disorder (ADHD), has increased dramatically. The reason for this phenomenon is that it is claimed that methylphenidate increases concentration and improves academic prowess, with unsubstantiated reports of increases in academic performance of up to $36 \%{ }^{2}$

Doctors are required to adhere to the Health Professions Council of South Africa (HPCSA) guidelines, according to which methylphenidate is a highly scheduled drug to be prescribed and dispensed only under strictly controlled conditions. On the other hand, there is pressure from the community to prescribe enhancement therapies in cases where there is no specific diagnosis present.

The following arguments reflect the concerns noted by various role-players:

- Using cognitive enhancement is a form of cheating, and it allows users an unfair advantage.

- It is dishonest and detracts from the user as role-model for others.

- The problem of indirect coercion - the belief that everybody else is taking these drugs and that I will be left behind if I do not.

- The argument that stimulants are dangerous - both because of direct physiological side-effects and the possibility that they are habit forming.

- If enhancement is not regulated or even banned outright, it will inevitably result in an eventual unknown future 'post-human' being, an unnatural entity with the expected potential to harm, abuse or suppress those who have not been exposed to the enhancement therapies. ${ }^{3,4}$

\section{Background}

Cognitive-enhancing drugs, or nootropics, have been around for a long time. Various cultures have used certain indigenous herbs to promote memory and concentration for thousands of years.

In conventional psychopharmacology, nootropics include psychostimulants such as methylphenidate, modafinil, amphetamines and medicinal caffeine. Other classes of non-stimulant nootropics include drugs used to treat dementia, such as donepezil, rivastigmine and galantamine. Despite evidence that methylphenidate improves domains of cognitive functioning, there is also evidence that in subjects with a high memory span and familiarity of tasks, functioning may actually deteriorate. ${ }^{5,6}$ The mere fact that there is some evidence of benefit would be sufficient reason to expect that the demand for methylphenidate for cognitive enhancement will continue.

\section{Safety concerns}

Methylphenidate is only registered for specific indications such as ADHD and narcolepsy, which implies that prescribing it to improve concentration in healthy students is done 'off-label'. Although 'offlabel' use is controversial in the medical fraternity, it is not illegal, and is often done in clinical practice.

The risk-benefit ratio must always be considered. Although this applies to all drugs, it is more relevant in 'off-label' prescriptions. It would not be considered justifiable to prescribe a dangerous drug to healthy subjects merely to increase their cognitive performance, especially if the evidence of efficacy is not conclusive.

Although methylphenidate is generally considered a safe and widely used drug, potential users must be made aware that 
cardiac complications have been reported in some high-risk groups.

\section{The question of addiction}

An emotive response often associated with the use of methylphenidate is the fear of addiction. Methylphenidate is chemically similar to cocaine and may have similar potential for abuse or addiction. In countries such as Sweden it was banned during the 1960s, but current evidence demonstrates that if it is used at prescribed doses, the risk of abuse is very low.

\section{Why enhancements may be needed}

Many physicians may respond with a 'gut feeling' that enhancement is wrong, but there are also clear reasons why it may be beneficial, for example: ${ }^{1,7}$

- The human propensity for violence and conflict may be lessened by enhancing capacities for impulse control, sympathy, altruism or moral imagination, through pharmaceutical or genetic interventions.

- Enhancement of the human capacity to extract nutrients from current foods, or even from substrates that we have been unable to use as food sources previously, may alleviate food shortages.

- Enhancement of the 'normal' viability of human gametes and/or embryos may be needed in an increasingly toxic environment to counteract a decrease in fertility and reduce the risk of lethal mutations.

- Enhancements may help us adapt physiologically to climate change and its associated dangers.

- Enhancement of the immune system to accelerate the development of resistance to virulent emerging infectious diseases.

- Cognitive enhancement may enable students in an age of information overload to cope with daily demands and their competitive academic environment.

- A speculative reason for cognitive enhancement could include the likelihood that it assists us to be more virtuous, as virtuous behaviour depends on sound judgements, and sound judgements depend on good ability to reason and process information. ${ }^{1}$

\section{Questions about cognitive enhancement \\ Is enhancement cheating?}

In most sports, performance-enhancing drugs are banned, as it is believed that those who use them have an unfair advantage. Sport guidelines are clear and 'punishment' for disobeying the rules is often severe - resulting in prolonged and/or permanent banishment from competition.

Nevertheless, enhancement by technological means is not seen as morally problematic, as long as it falls within the rules. This raises the issue as to whether the act of cheating is determined by the rule and not the morality of the act in itself.

\section{What would the good doctor do?}

The good doctor may experience a dilemma in deciding what the right course of action would be regarding cognitive enhancement. What good doctor would stand in the way of a student fulfilling his or her potential with all the resulting benefits? On the other hand, might this student be at risk of becoming addicted to methylphenidate?

The physician has an obligation to prevent, cure or reduce suffering - but there is no established duty to make patients 'better than well. ${ }^{8}$ It may therefore also be argued that the virtuous doctor's responsibility ends with the rational and ethical treatment of disease or an established disorder. Nevertheless, although enhancement may fall outside the doctor's scope of practice, he or she has been awarded the custodianship thereof.

Even if enhancement is not considered a 'proper' medical outcome, is enhancement itself morally permissible or not? This speaks directly to the ambivalence experienced by many medical practitioners in the debate around the use of methylphenidate in healthy students.

\section{Who should decide?}

The paternalistic doctor role is no longer seen as the only model of interaction between patient and doctor. Informative and deliberative styles of interaction are now considered more appropriate. Doctors may claim that their decisions on whether or not to prescribe cognitive enhancement are purely based on safety concerns and a lack of proven efficacy. But in truth, decisions are also based on the doctor's own opinion regarding the ethics of enhancement. This would be considered a paternalistic action on behalf of the doctor and the justification of enhancement should actually not fall under the jurisdiction of medicine per se.

\section{What about justice?}

Access to methylphenidate would be unequal in South Africa. Availability of a doctor, financial means and knowledge about enhancement are not equally available. Various technologies and interventions are widely used to promote academic achievement, however, from the simple use of artificial light to computers and the Internet. It would be unthinkable to ban these technologies because inequalities in availability prevent some from access.

\section{Should we worry about the slippery slope?}

The slippery slope argument states that if an individual or society embarks on a certain course of action, it is likely to result in a chain of consequences. The end of this chain is highly immoral or undesirable and implies the development of a so-called post-human state. The only way to prevent the end result is not to start the process at all. ${ }^{9,10}$ But the slippery slope argument may also be considered a continuum fallacy, which states that there is a mistake in logic in that the eventual outcome is not a given.

Responsible development of enhancement technologies would be expected to have various checks and balances built into the process. There is also no clear reason why a cognitively enhanced being would be less moral or ethical.

\section{Is enhancement against nature?}

The 'argument from nature' is commonly used in contemporary debate on moral issues in bioethics. This is a conservative stance, condemning anything that is perceived as being against what is seen as 'natural. But man has been modifying nature for thousands of years in the process of enhancing quality of life and to ensure safety. Nature has given us illnesses like malaria, cancer and AIDS, and to claim that we have no moral right to fight these illnesses because they are determined by nature is clearly irrational.

\section{Should doctors restrict themselves to therapy?}

Therapy - an intervention aimed at the treatment of disease would generally not be considered to be an unethical practice, whereas enhancement is clearly frowned upon in various quarters. ${ }^{1}$ A consistent distinction between therapy and enhancement is difficult to uphold ${ }^{10-12}$ when considering that an intervention such as vaccination is not aimed at treating a disorder or disease. As it 
is in fact aimed at enhancing the immune system, this argument becomes less clear.

\section{Is erosion of character not inevitable?}

The expressivist character concern states that the pursuit of enhancement, independently of the eventual outcomes and consequences, is a sign of an already existing 'bad' character. The consequentialist concerns predict that the active pursuit of enhancement leads to the progressive development of bad character. Many 'bad' decisions and actions will eventually contribute to the failure of character integrity.

Bioconservatives argue that there is something intrinsically valuable about human nature, and if we enhance ourselves, this will be lost. However, it is highly problematic to think of 'human nature' as a moral concept or a moral desideratum. If 'human nature' is, morally speaking, something to preserve at all costs, we must accept that we ought also to preserve all the characteristics which are part of 'human nature', such as jealousy, vindictiveness and cruelty, that are generally regarded as (also morally) undesirable.

Sandel claims that the pursuit of enhancement betrays and exacerbates existing character deficits because it demonstrates a lack of appreciation for 'giftedness' - a sense of accepting the limitations of human powers and what we cannot control. ${ }^{13}$ Those lacking an appreciation for 'giftedness' and who promote the processes of enhancement do so because of a desire for mastery and perfection. This relates to a Promethean aspiration to remake nature, purely to serve our own purposes and satisfy our desires.

It is not clear how the jump from a desire to be better or live longer to the desire to master others or the desire for immortality can be made. Sandel's argument is furthermore inconsistent in that he accepts that we ought to fight disease, whereas disease is undoubtedly part of the 'giftedness of life. Sandel's alleged distinction between those aspects of life that we ought to 'accept' in children, and those we wish to 'transform' (also through education), is quite unclear.

If we consider humans today to be more advanced than our predecessors of a few centuries ago, whether because of use of new technologies, better nutrition or whatever, are we less moral today than in 1800 ? Interactions between humans remain fraught with old and new immoralities, but there are also many examples of altruism and enhanced moral arguments put forward by the very societies that promote enhancement.

\section{Conclusion}

Whether the use of methylphenidate by healthy students should be allowed or not is not straightforward. However, the demands by users and ongoing developments in cognitive enhancement are rapidly leading us to the point where not to decide is in fact to decide. ${ }^{8}$ Further developments in cognitive enhancement may well be safer than methylphenidate, but this does not imply that the use of cognitive enhancement techniques should be put on hold until then. Nevertheless, some doctors either remain unconvinced by the arguments for the promotion of cognitive enhancement in healthy subjects or refuse to debate the issue.

This anti-enhancement point of view may be derived from a combination of 'fear of doing wrong' and traditional paternalism. But the demand for enhancement requires debate broader than that of traditional medicine, and medical practitioners should be seen as participants in this debate rather than all-powerful gatekeepers.

Finally, we would argue that, before the prescription of performance-enhancing drugs is considered as standard practice, more decisive clinical trials about their efficacy and safety for this purpose are required.

1. Buchanan AE. Beyond Humanity?: The Ethics of Biomedical Enhancement. Oxford: Oxford University Press, 2011. [http://dx.doi.org/10.1093/acprof:oso/9780199587810.001.0001]

2. Delport S. Ritalin se nuwe (onwettige) gebruike. Rooi Rose 2011. http://www.rooirose.co.za/index. php?p[IGcms_nodes][IGcms_nodesUID]=45a4941c2e9f8e466e99748e9db8fb00 (accessed 10 May 2011).

3. Fukuyama F The world's most dangerous ideas: Transhumanism. Foreign Policy 2008;5:42-43.

4. Wikler D. Paternalism in the age of cognitive enhancement: do civil liberties presuppose roughly equal
4. Wikler D. Paternalism in the age of cognitive enhancement: do civil liberties presuppose roughly equa
mental ability? In: Savulescu J, Bostrom N, eds. Human Enhancement. Oxford: Oxford University Press, 2010:341-356.

5. De Jongh R, Bolt I, Schermer M, Olivier B. Botox for the brain: enhancement of cognition, mood and pro-social behavior and blunting of unwanted memories. Neuroscience \& Biobehavioural Review 2008:32:760-776.

6. Geppert CMA, Taylor PJ. The religion of benzodiazepines. Psychiatric Times 2007;24(4). http://www. psychiatrictimes.com/print/article/10168/54151 (accessed 1 June 2012).

7. Harris J. Enhancing Evolution. Princeton, NJ: Princeton University Press, 2007.

8. Drabiak-Syed K. Physicians prescribing 'medicine' for enhancement: Why we should not and cannot overlook safety concerns. Am J Bioeth 2011;11(1):17-19. [http://dx.doi.org/10.1080/15265161 2010. 534535]

9. Den Hartogh G. The slippery slope argument. In: Kuhse H, Singer P, eds. A Companion to Bioethics. Oxford: Blackwell, 1998:280-292.

. Dane, $N$. Bostrom N, eds. Human Enhancement. Oxford: Oxford University Press, 2010:25-42. 1. Harris, J. Enhancement as a moral obligation. In: Savulescu J Boston Oxford: Oxford University Press, 2010:131-154.

12. Holtug, N. Creating and patenting new life forms. In: Kuhse H, Singer P, eds. A Companion to Bioethics. Oxford: Blackwell, 1998:206-214

13. Sandel MJ. The case against perfection: ethics in the age of genetic engineering. Cambridge, Mass. Belknap Press of Harvard University Press, 2007.

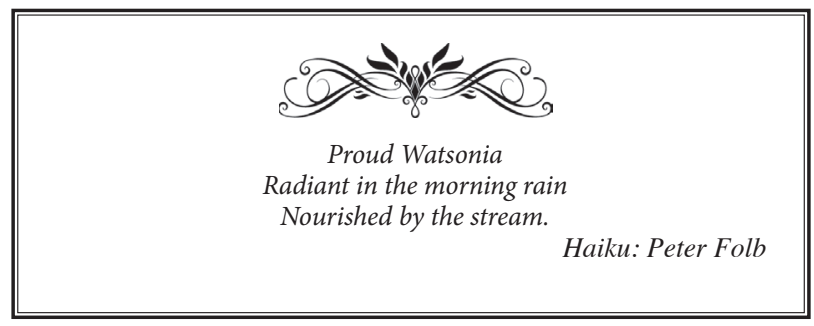

